\title{
Amathonte : les abords Sud-Ouest de l'agora - 2018
}

Ludovic Thély, Julien Adam, Camille Castres, Antigone Marangou et Cécile Rocheron

\section{OpenEdition \\ Journals}

Édition électronique

URL : http://journals.openedition.org/baefe/1268

DOI : $10.4000 /$ baefe. 1268

ISSN : 2732-687X

Éditeur

ResEFE

\section{Référence électronique}

Ludovic Thély, Julien Adam, Camille Castres, Antigone Marangou et Cécile Rocheron, « Amathonte les abords Sud-Ouest de l'agora - 2018 » [notice archéologique], Bulletin archéologique des Écoles françaises à l'étranger [En ligne], Chypre, mis en ligne le 26 novembre 2020, consulté le 15 décembre 2020. URL : http://journals.openedition.org/baefe/1268 ; DOI : https://doi.org/10.4000/baefe.1268

Ce document a été généré automatiquement le 15 décembre 2020.

\section{(c) $(1)$}

Le Bulletin archéologique des Écoles françaises à l'étranger est mise à disposition selon les termes de la Licence Creative Commons Attribution - Pas d'Utilisation Commerciale - Pas de Modification 4.0 International. 


\section{Amathonte : les abords Sud-Ouest de l'agora - 2018}

Ludovic Thély, Julien Adam, Camille Castres, Antigone Marangou et Cécile Rocheron

\section{NOTE DE L'AUTEUR}

Autorité nationale présente : Yiannis Violaris (Département des Antiquités de Chypre, District de Limassol)

Composition de l'équipe de terrain : Ludovic Thély, responsable d'opération ; Julien Adam, chef de secteur ; Camille Castres, architecte ; Antigone Marangou et Cécile Rocheron, céramologues.

Partenariats institutionnels : École française d'Athènes, Ministère de l'Europe et des Affaires étrangères.

Établissements porteurs de l'opération : École française d'Athènes, Ministère de l'Europe et des Affaires étrangères.

Remerciements : La mission exprime sa reconnaissance à Marina Solomidou-Ieronymidou, directrice du Département des Antiquités de Chypre, et à Yiannis Violaris, archéologue responsable du district de Limassol, pour leur collaboration et confiance.

Données scientifiques produites :

Mission française d'Amathonte par l'EFA

Mission française d'Amathonte par l'Ambassade de France à Chypre

Amathonte 2018 


\section{Introduction}

1 L'École française d'Athènes et le Ministère de l'Europe et des Affaires étrangères soutiennent depuis 2014 la reprise des travaux archéologiques à Amathonte. Dans la partie encaissée de la ville antique, entre l'agora et le port extérieur submergé, la zone du prétendu bassin intérieur fait l'objet d'une exploration archéologique systématique, menée par une équipe internationale dirigée par Ludovic Thély (fig. 1)1. Les résultats des quatre premières campagnes ont permis de jeter un regard nouveau sur l'occupation de cette partie du site. L'objectif initial était de répondre à une problématique ancienne : existait-il à Amathonte un port intérieur aménagé ?

Fig. 1. Implantation des secteurs et des sondages, 2014-2018.

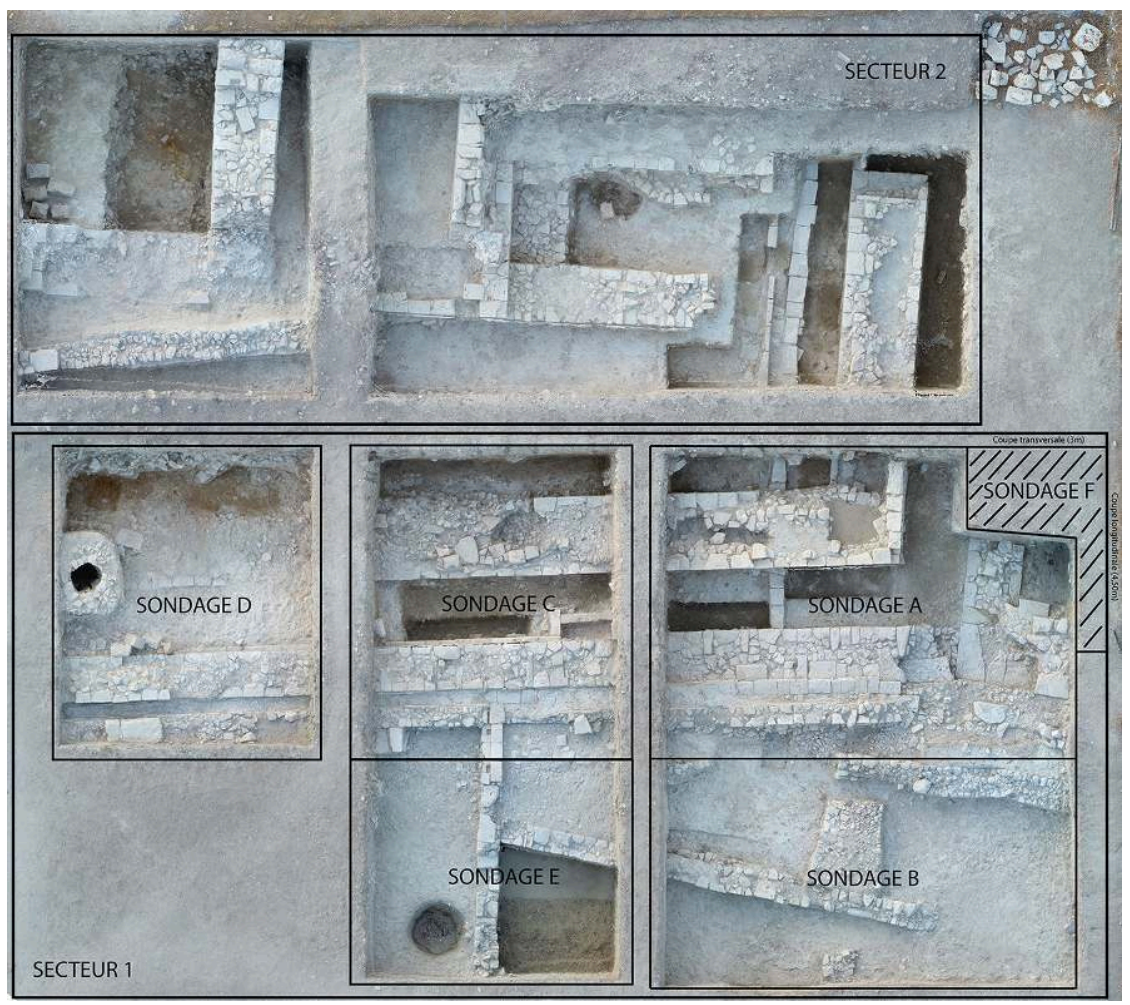

L. Thély, J. Adam / EFA.

2 L'enquête géomorphologique ${ }^{2}$ a permis de mettre en évidence trois grandes unités stratigraphiques :

- Unité A. Cette unité, la plus profonde, est constituée de sable de plage plus ou moins fin, parfois entrecoupé de passes de galets. Il s'agit des vestiges d'une paléoplage, qui pourrait être antérieure ou contemporaine de l'occupation du site.

- Unité $B$. Elle est constituée d'un important niveau argileux plus ou moins riche en matière organique. Ces dépôts sont clairement marins et la malacofaune y a été récupérée in situ. Cette unité trahit une dynamique sédimentaire très faible: ce sont des dépôts de décantation.

- Unité C. Plus épaisse que les deux autres, elle est composée de limons argilo-sableux beiges. La présence de cailloux et de fragments de céramique en grand nombre doit sans doute être interprété comme une couche de comblement. 
Les édifices monumentaux, repérés dans le Nord de la zone, ont été identifiés comme une succession de pièces de stockage dont l'ensemble forme un entrepôt de grande taille ouvert vers le Nord, sur l'une des voies principales d'accès à l'agora.

4 Le phasage chronologique des vestiges, rendu difficile d'interprétation par l'absence de matériel en place, peut être défini synthétiquement de la manière suivante :

- Phase A. Les vestiges antérieurs à l'époque hellénistique, dans les couches les plus profondes, témoignent d'une occupation ancienne de la zone du bassin intérieur. Il est vraisemblable que cet espace ait été occupé dès l'époque classique, peut-être même à l'époque archaïque.

- Phase B. La construction, au début de l'époque impériale (ou à la toute fin de l'époque hellénistique ?), de structures massives rendent compte d'un réaménagement de la zone, peut-être à la suite d'un tremblement de terre (certains sont connus par la littérature antique). Une monumentalisation des espaces commerciaux, en lien avec les aménagements constatés sur l'agora, semble ainsi se dessiner nettement.

- Phase $C$. Le remblaiement des structures, au III $^{\mathrm{e}}$ siècle de notre ère, a laissé place à des activités artisanales limitées, notamment au Sud de la zone explorée où le matériel découvert (monnaies, céramiques) présentent un caractère tardif.

- Phase $D$. La phase précédente s'est très vraisemblablement accompagnée d'une phase de spoliation des vestiges. De nombreux blocs ont été prélevés, ce dont témoignent à la fois la perte de plusieurs assises ainsi que les perturbations très nettes enregistrées dans la stratigraphie.

Enfin, la très grande variété du matériel mis au jour donne à voir des productions très nombreuses: céramique fine, culinaire et commune; amphores de transport; monnaies (quatre-vingt-deux à ce jour) ; fragments moulurés d'édifices hellénistiques ; terres cuites; lampes, etc. L'arc chronologique est très large : il court du ChyproArchaïque à l'époque protobyzantine.

\section{Études de matériel}

\section{Étude amphorologique (fig. 2-3)}

6 Une étude amphorologique menée par Antigone Marangou (Université de Rennes 2) s'est tenue en juillet 2018. Le tri, l'identification et la réalisation des dessins des amphores mises au jour en 2017 ont confirmé les hypothèses suggérées par l'étude de stratigraphie. Les US de remblais antiques contiennent un matériel mélangé, dont le terminus est donné par des amphores tardives (notamment celles dites à «anses de panier ", datées du $\mathrm{v}^{\mathrm{e}}$ siècle de notre ère). Les US plus profondes, qui correspondent aux différents niveaux d'occupation ou de fondation des édifices, présentent un matériel plus homogène : fin de l'époque hellénistique ou du Haut Empire.

7 Le catalogue des timbres amphoriques s'est considérablement enrichi à l'occasion de la campagne 2017. Ce sont plus d'une centaine de timbres complets ou fragmentaires qui composent désormais le catalogue. Ils présentent deux intérêts : Ils contribuent d'abord à la datation des US et donc des structures associées; certains sont des unici, qui viennent enrichir les catalogues existants et permettent de réviser les datations communément admises. 
Fig. 2. Amphore pamphylienne complète restaurée en 2017.

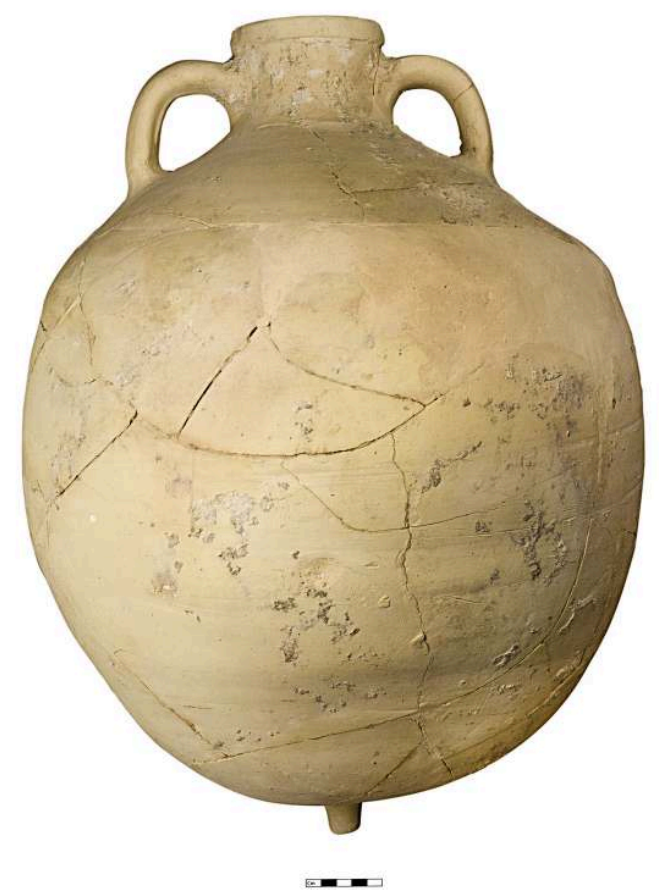

A. Athanassiou / EFA.

Fig. 3. Timbre amphorique.

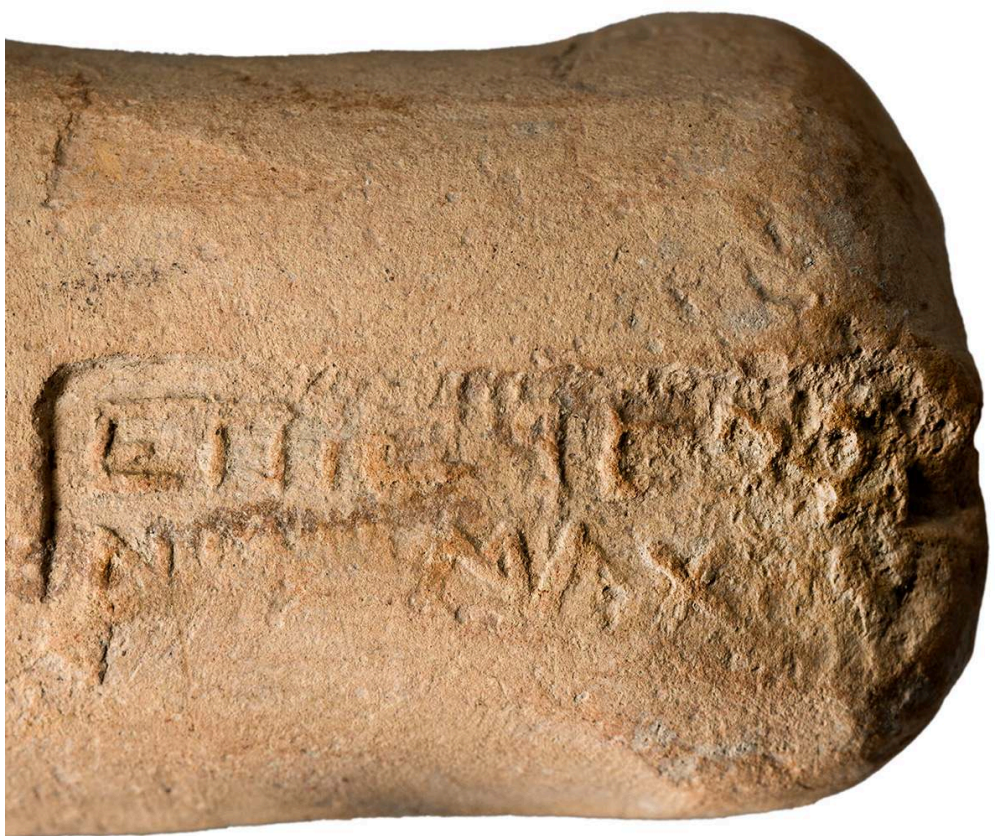

A. Athanassiou / EFA. 


\section{Campagne photographique}

Le photographe du musée de Limassol, Athanassos Athanassiou, a pris en charge le traitement photographique du matériel mis au jour depuis 2014 à des fins de publication. Trois-cents objets ont ainsi été photographiés (monnaies, lampes, terres cuites, timbres amphoriques et objets lithiques).

\section{Relevés architecturaux (fig. 4-6)}

Camille Castres a achevé les relevés (coupe et photogrammétrie) des blocs d'architecture moulurés découverts dans les couches de remblais. Ces fragments appartiennent à plusieurs séries de bâtiments qui devaient se trouver sur l'agora d'Amathonte : ils donnent un aperçu des constructions hellénistiques.

En outre, les relevés des structures architecturales mises au jour en 2017 dans le Sect. 02 (entrepôts), ainsi que les murs dégagés en 2018 (Sect. 01, sondage F) ont été achevés. Le plan général du site est désormais établi, de même que les coupes Est-Ouest et Nord-Sud. Enfin, les élévations de l'UC 1005 et 2005 ont également été achevées.

Fig. 4. Relevé architectural du site.

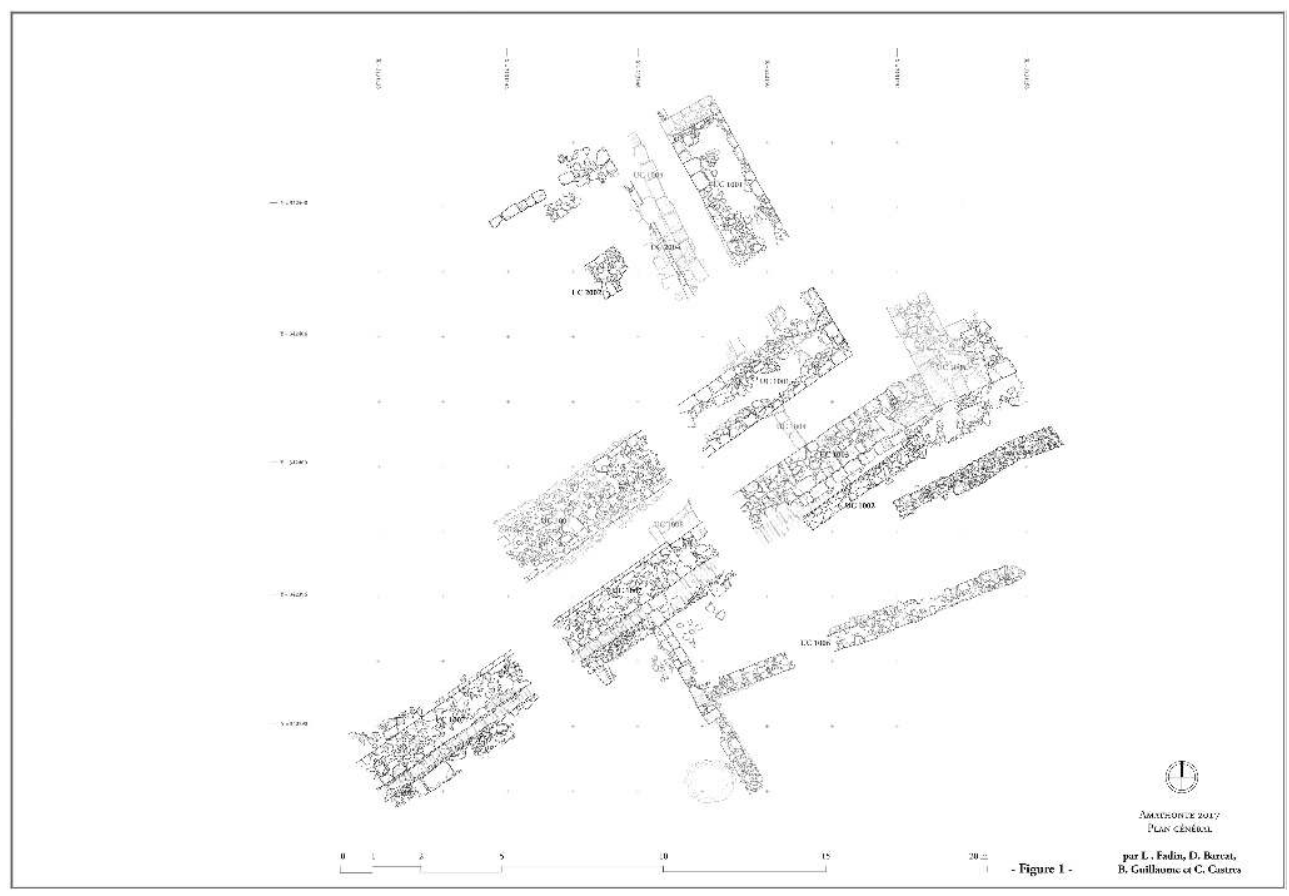

C. Castres / EFA. 
Fig. 5. Relevé architectural d'un bloc mouluré.

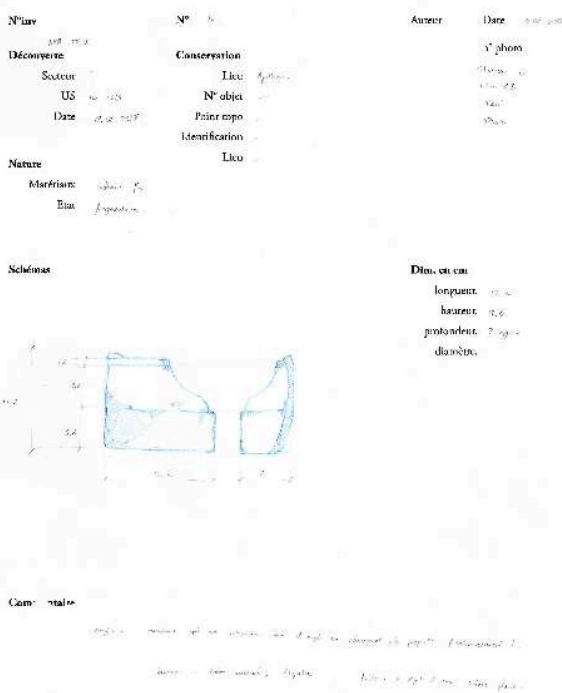

C. Castres / EFA.

Fig. 6. Orthophotographie des structures mises au jour.

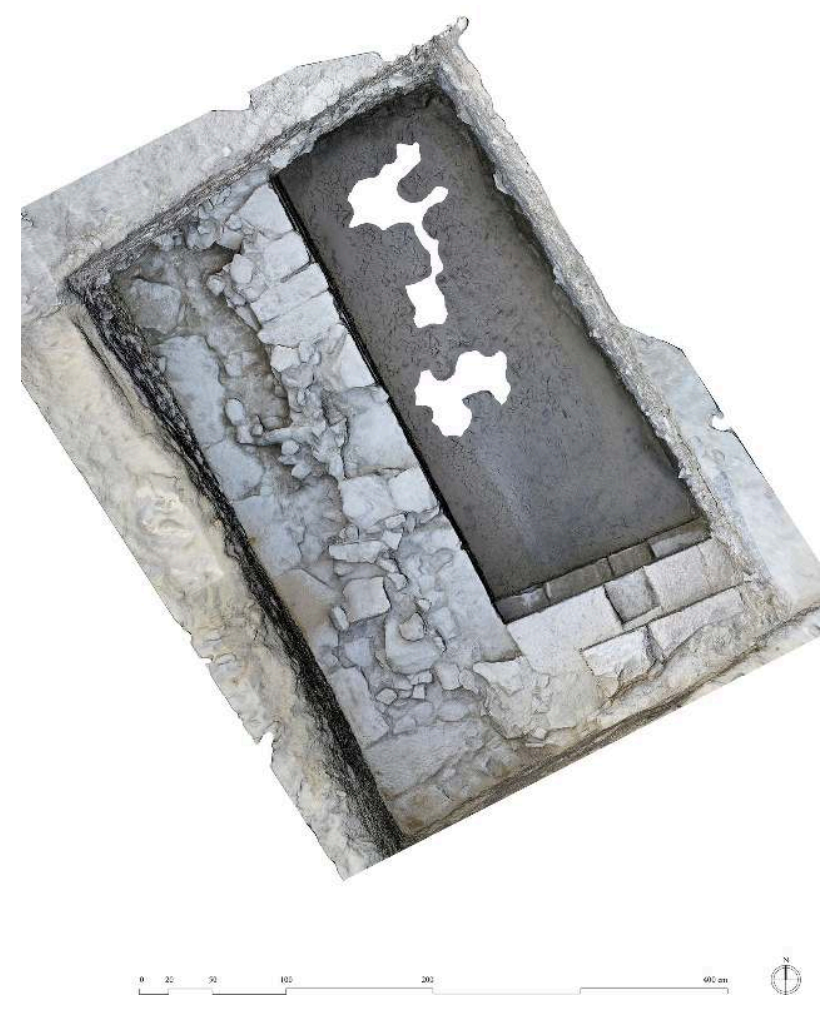

J. Adam, C. Castres / EFA.

Bulletin archéologique des Écoles françaises à l'étranger , Chypre 


\section{Étude des monnaies (fig. 7)}

11

Grâce à la diligence du Département des Antiquités de Chypre, les monnaies découvertes en 2016 ont été restaurées. Sur les vingt-neuf qui ont été étudiées et photographiées, ving-trois sont identifiables. Elles s'étendent sur une période chronologique large, des souverains ptolémaïques à l'empereur Honorius (395-423).

Fig. 7. Monnaie de Gordien III.

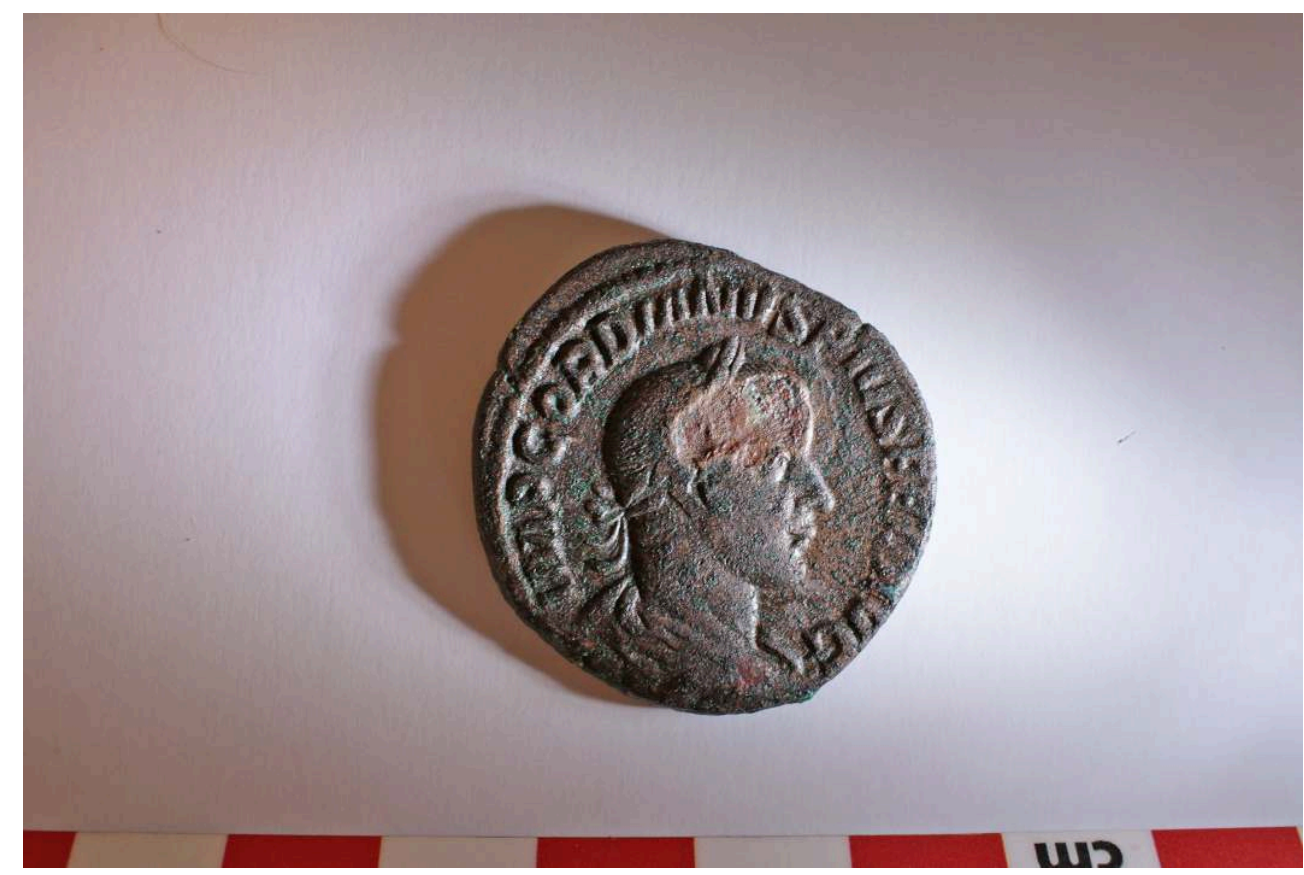

J. Adam / EFA.

On distingue trois séries principales :

1. Les monnaies lagides, découvertes dans les US les plus profondes, et qui confirment les autres éléments de datation.

2. Les monnaies impériales.

3. Les monnaies des $\mathrm{III}^{\mathrm{e}}-\mathrm{IV} \mathrm{V}^{\mathrm{e}}$ s., dont la concentration dans le sondage $\mathrm{E}$ impose la conclusion suivante : l'extrémité Sud de la zone urbanisée a connu, à partir du III siècle, un nouvel aménagement et l'apparition de nouvelles activités artisanales.

\section{Étude des terres cuites (fig. 8)}

13 La dernière partie de la mission d'étude a consisté en une analyse des figurines de terre cuite. Aux quarante objets mis au jour de 2014 à 2017, se sont ajoutés trois fragments remarquables découverts lors de la campagne 2018: une tête d'Aphrodite, un buste d'équidé d'époque Chypro-Archaïque et un fragment de visage féminin ChyproArchaïque de grande taille. 
Fig. 8. Fragment de statuette en terre cuite.

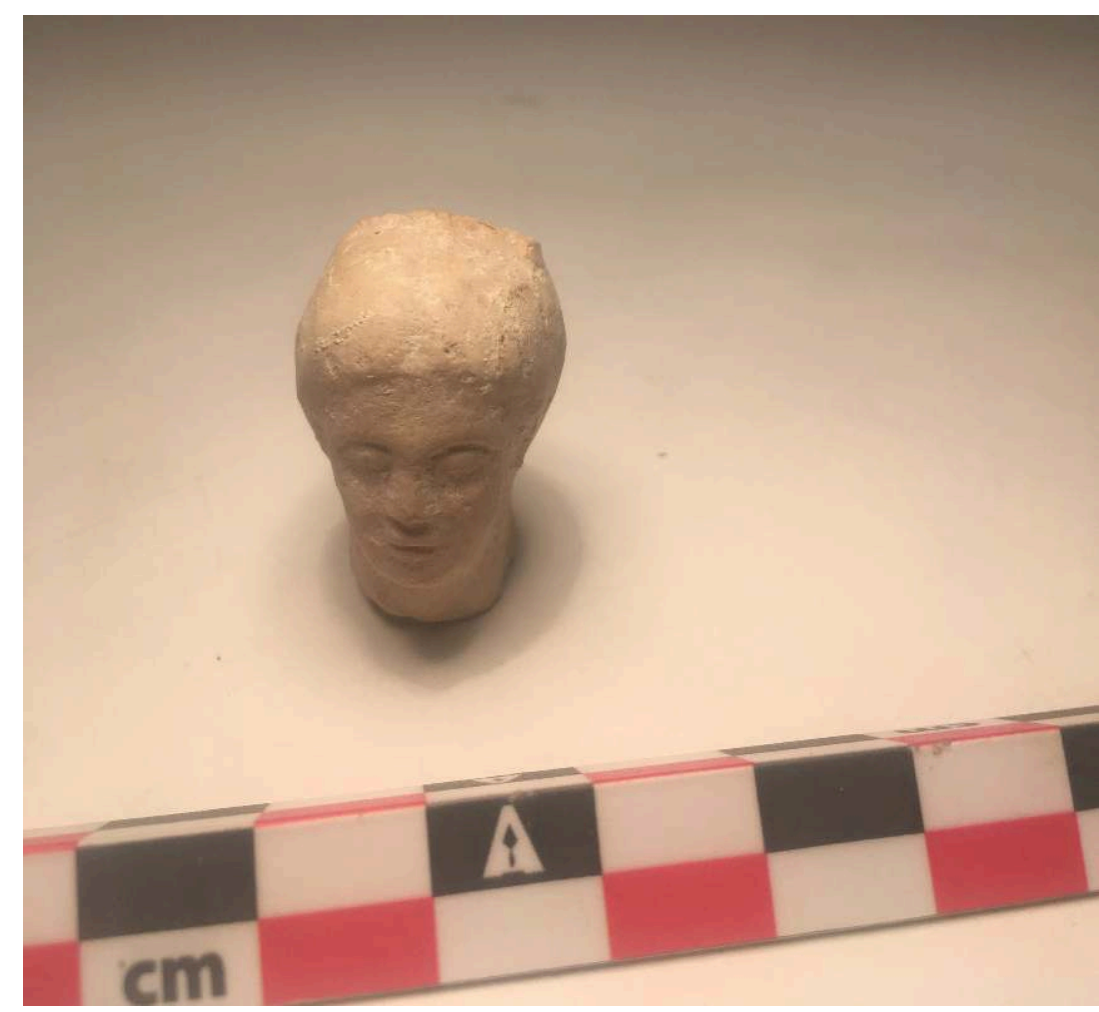

L. Thély / EFA.

\section{Recherches de terrain}

La campagne 2018 s'est concentrée sur le dégagement de la structure UC 1005 découverte en 2014, puis fouillée de manière systématique en 2017. Une portion d'un mur monumental avait ainsi été dégagée sur moins d'un mètre. Il présentait un appareil différent des structures connues dans la zone jusqu'ici. Surtout, du point de vue de la stratigraphie, il était associé à une couche de sable marin tel qu'on en trouve nulle part ailleurs dans les autres sondages.

Il nous est apparu nécessaire de poursuivre l'exploration de cette zone dans la mesure où la présence de sable, contenant un matériel sans doute archaïque, pouvait permettre de répondre à la problématique initiale, celle de la présence d'un port aménagé au SudOuest de l'agora et à proximité des édifices de stockage.

Nous avons ainsi opéré l'implantation d'un sondage de $4,5 \times 3 \mathrm{~m}$, dans la continuité de l'UC 1005 légèrement dégagée en 2017.

\section{Les données de la stratigraphie (fig. 9-11)}

17 La séquence stratigraphique du sondage $\mathrm{F}$ débute par une US de surface au matériel très mélangé (US 1501). Elle est suivie par deux situations très différentes dont la limite est clairement visible dans la coupe. En effet, il s'avère qu'une tranchée (US 1506) a été creusée dans l'alignement de l'UC 1012 à une période encore indéterminée, afin d'en prélever les blocs. Le remplissage de cette tranchée comporte d'abord les US 1503 
et 1507, deux matrices brunes relativement meubles aux inclusions charbonneuses et petits galets noirs. Elles sont suivies par l'US 1508, un remblai limono-sableux de couleur brune et très meuble comportant du matériel brûlé ; puis par l'US 1510, une argile brune très compacte présente sur une petite dizaine de $\mathrm{cm}$. Par leur épaisseur importante, les deux US suivantes composent la majeure partie du remplissage. La première (US 1512) est argilo-limoneuse, de couleur brune et compacte. Elle comprend du matériel brûlé en abondance, de nombreux fragments d'enduit hydraulique, mais aussi quelques "patchs» d'oxyde de fer. La deuxième est plus meuble, composée de limon et de sable (US 1515). On y remarque encore la présence d'oxyde de fer, d'enduit hydraulique, auquel s'ajoutent cette fois quelques fragments de mortier. C'est également lors de la fouille de cette US que furent découverts plusieurs fragments de bassins qu'il convient de mettre en relation avec la phase artisanale déjà repérée les années précédentes. Enfin, on note à cet endroit un léger surcreusement de la tranchée qui témoigne sans doute du dégagement des blocs pour en faciliter le prélèvement.

Cette tranchée que nous venons de décrire, traverse assez nettement une séquence stratigraphique localisée à l'Est du sondage. Celle-ci débute avec l'US 1504, une matrice blanche/rose, déjà rencontrée en 2014 (US 1008), qui se compose manifestement d'un mélange de mortier et de calcaire sur une dizaine de cm. Elle est suivie par l'US 1509, un niveau de pierres irrégulière de calibre moyen mêlées à une matrice argileuse compacte aux nuances grises/vertes. Sous cette dernière apparait une matrice argilosableuse de même couleur, mais comprenant, cette fois, des pierres irrégulières de petit calibre ainsi que quelques inclusions de charbon et d'oxyde de fer. Nous poursuivons avec l'US 1516, une strate de cailloutis mêlée à une matrice argilo-sableuse de couleur jaune, dont la fouille a permis la découverte d'une tête de statuette féminine en terre cuite. Cette US est traversée par une fosse (US 1514), remplie par une matrice argileuse et compacte de couleur noire, à l'intérieur de laquelle furent mis au jour de nombreux fragments de tuiles. Sous ces niveaux apparait finalement la couche de sable noire, déjà repérée l'année précédente. Nous avons pu la fouiller sur une profondeur d'1,20 m. Elle contient de nombreux tessons roulés par la présence supposée d'eau en mouvement à cet endroit et s'appuie sur les UC 1005 et 1012. 
Fig. 9. Coupe stratigraphique Est-Ouest.

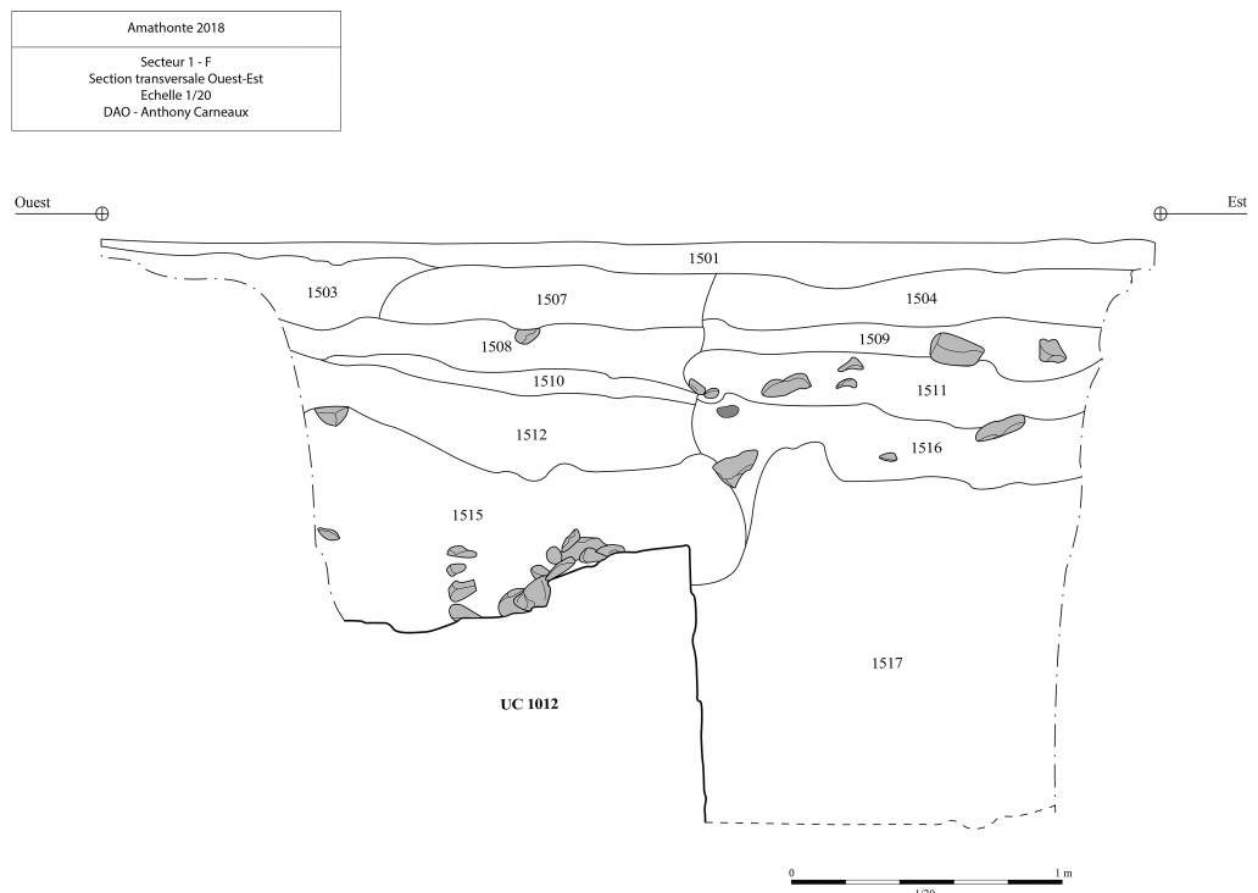

A. Carneaux / EFA.

Fig. 10. Coupe stratigraphique Nord-sud.

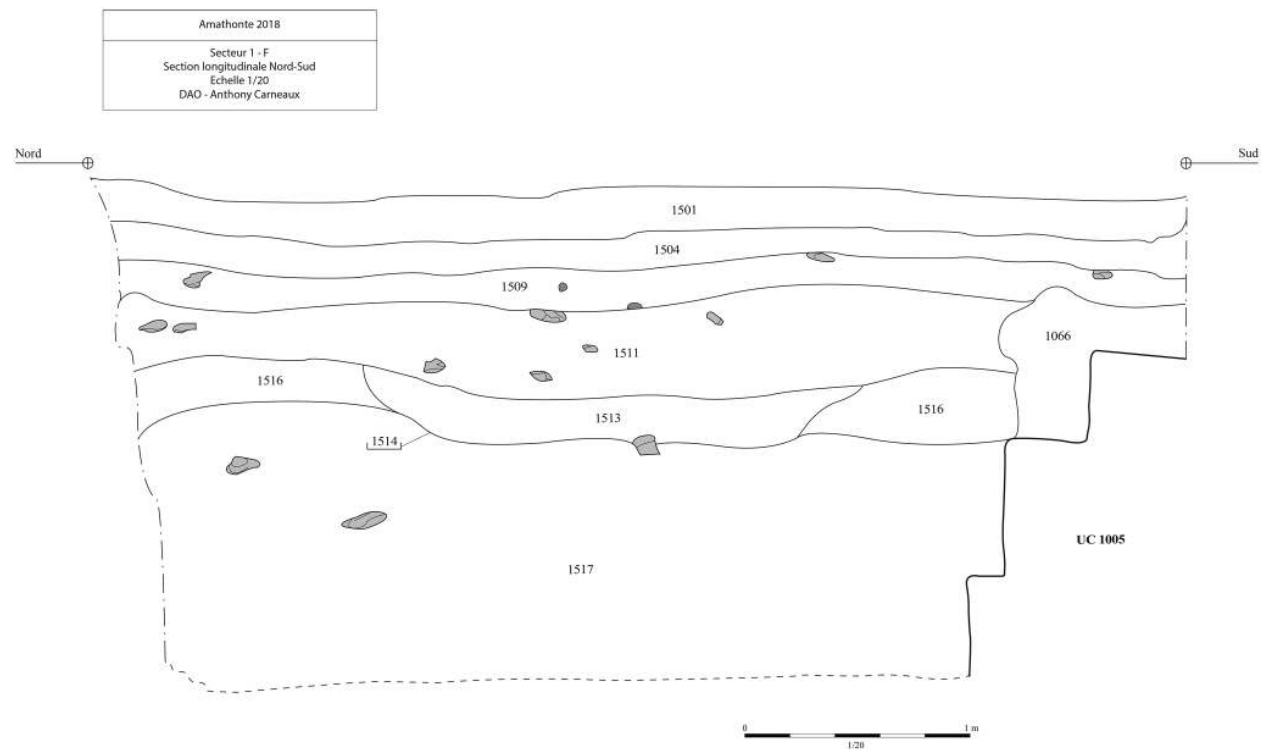

A. Carneaux / EFA. 
Fig. 11. Photographie de l'US $1516 \& 1517$.

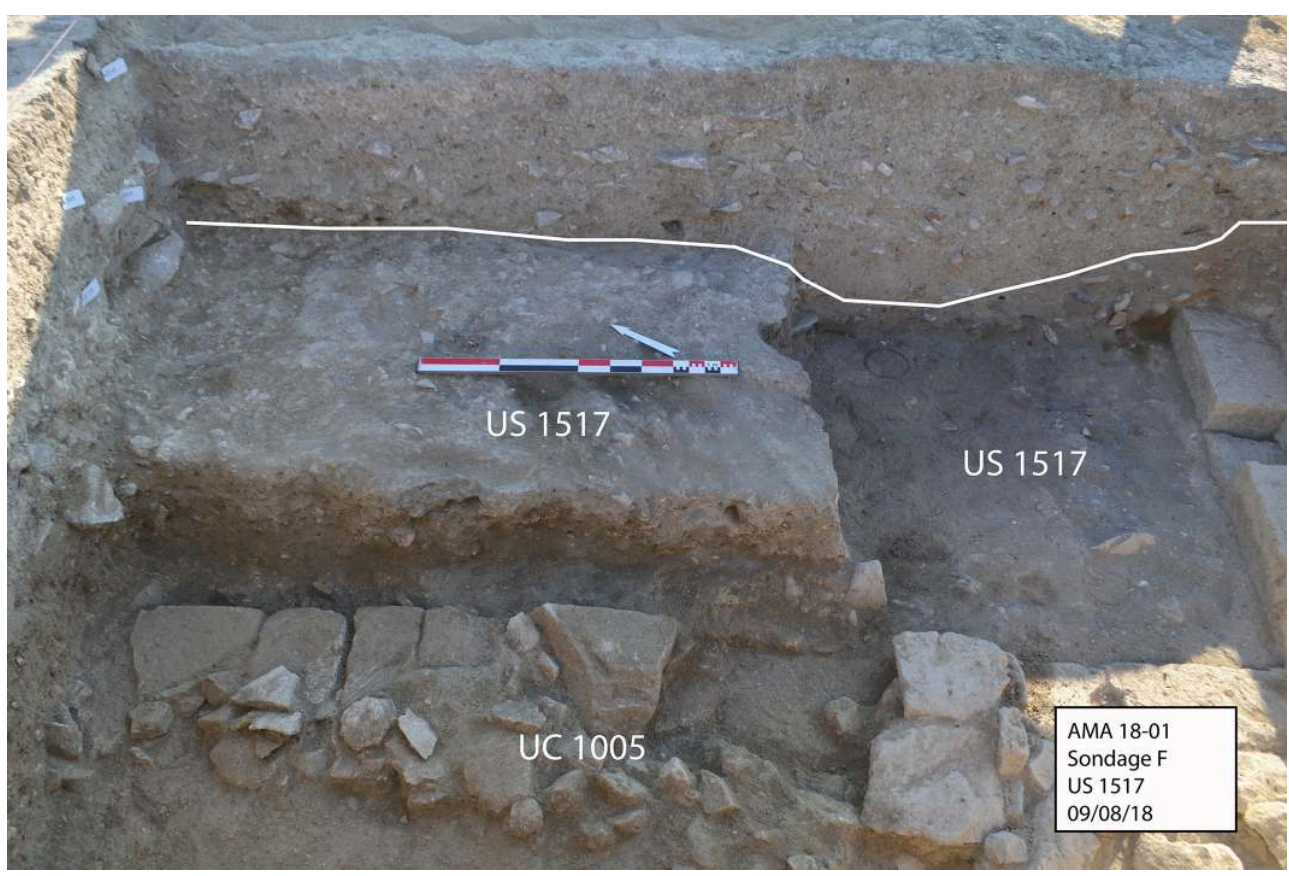

J. Adam / EFA.

\section{Les vestiges architecturaux (fig. 12-14)}

19 La fouille a permis le dégagement sur la totalité de la longueur et de la largeur du sondage de deux murs.

20 UC 1005. Il s'agit d'un mur en maçonnerie de parement et remplissage, en grand appareil, mis au jour sur quatre assises, dont l'une affleure à peine sous l'eau. Le niveau de fondation ne semble pas avoir été atteint. Seul le parement Nord a été rendu visible par la fouille. De ce côté, chaque assise mise au jour est en retrait par rapport à la précédente, on peut ici parler de mur à degrés. Ces retraits font, de bas en haut, approximativement 11,18 et $38 \mathrm{~cm}$. Le dernier, bien plus profond, témoigne peut-être d'un remaniement du mur. Les assises ont une hauteur, de bas en haut de 49,5, 58 et $42 \mathrm{~cm}$. La surface de la première assise, aujourd'hui noyée sous de l'eau douce semble en partie couverte de de concrétions, peut-être liées à un séjour prolongé dans l'eau de mer. Un des blocs de la troisième assise présente une cavité parallélépipédique de 13 x 29,5 cm taillée à son angle supérieur Est. Elle est rendue entièrement visible par le retrait de l'assise supérieure. Un petit bloc vient la boucher à une profondeur d'approximativement $29 \mathrm{~cm}$. Le retrait de la dernière assise nous permet aussi d'apercevoir la typologie des blocs des première assises. Ils semblent être en retour d'équerre ou en retour d'équerre et démaigrie à queue rabattue. Les faces de parement sont très bien taillées, les joints fins. Le parement nous montre une construction qui paraît être en carreau et boutisse ou panneresse et boutisse. Ce mur, à l'exception peutêtre de l'assise la plus haute, ne semble pas composée de blocs en remploi.

UC 1012. Sur l'UC 1005 s'adosse l'UC 1012. Les deux ont pu fonctionner de manière contemporaine. L'UC 1012 est en maçonnerie de parement et remplissage, en grand appareil sur trois assises, puis en moyen appareil pour la plus haute pour le parement Est. On observe un arrachement des blocs les plus hauts, ce qui correspond à la coupe 
stratigraphique, probablement afin de remployer ces blocs dans une autre construction. Seul le parement Est a pu être dégagé. Il présente une élévation conservée de quatre assises, la première est à moitié noyée sous l'eau. Ensuite, chacune d'elle présente une hauteur, de bas en haut, de 32,5, 40 et entre 20 et $23 \mathrm{~cm}$ pour la dernière. L'arrachement des dernières assises du mur nous permet de voir la typologie des blocs. Pour les assises basses, ils sont en retour d'équerre et à queue rabattue, les faces de parement sont bien taillées et les joints fins. On note par ailleurs des traces d'outils sur certaines faces de parement. La dernière assise est plus irrégulière et dans la taille des blocs, et dans les joints parfois fourrés de petites pierres. Tout semble indiquer là encore que la construction, au moins sur ces trois premières assises, n'est pas composée de remplois.

Fig. 12. Relevé architectural de l'UC 1012.

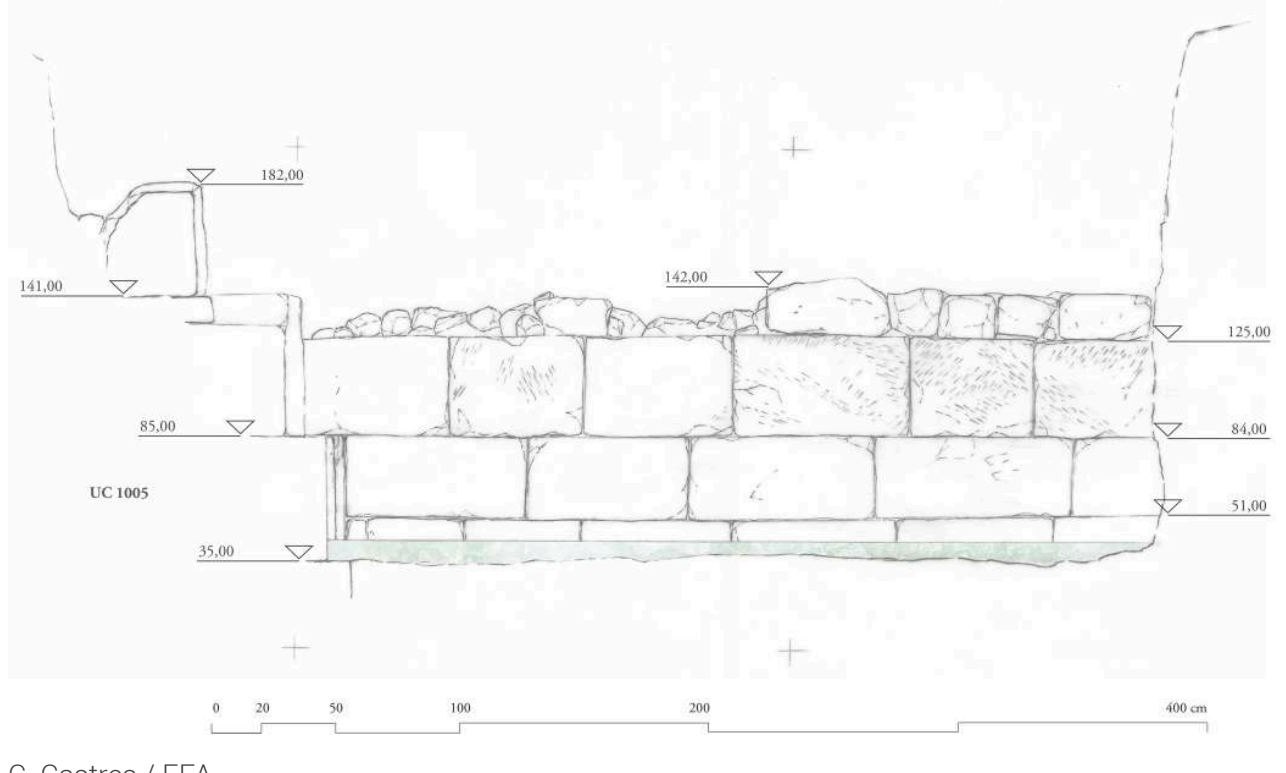

C. Castres / EFA 
Fig. 13. Photographie de détail des blocs de l'UC 1005.

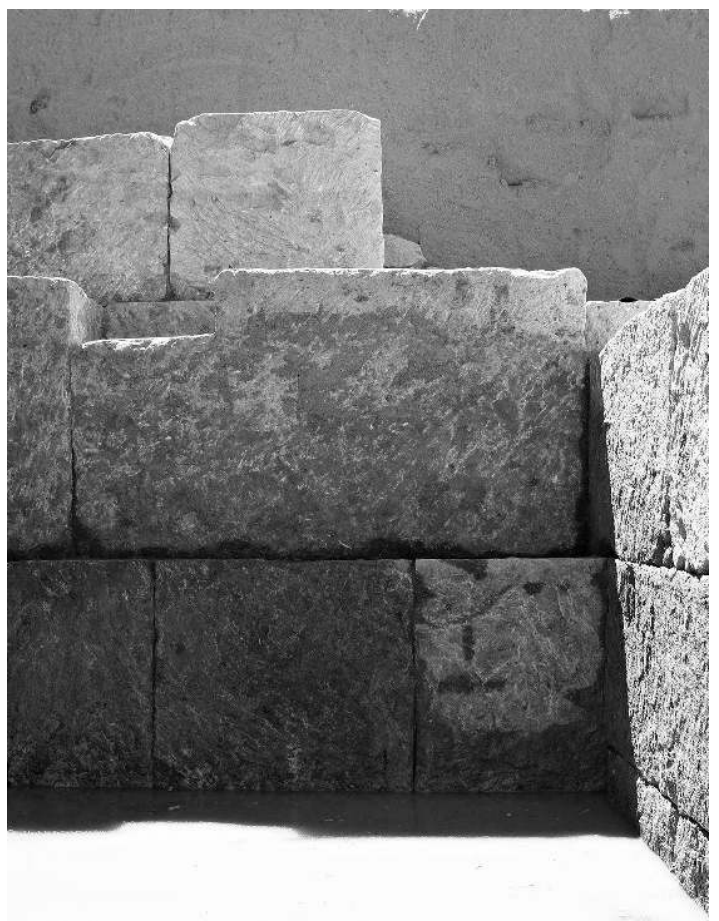

C. Castres / EFA

Fig. 14. Photographie de détail des blocs de l'UC 1005.

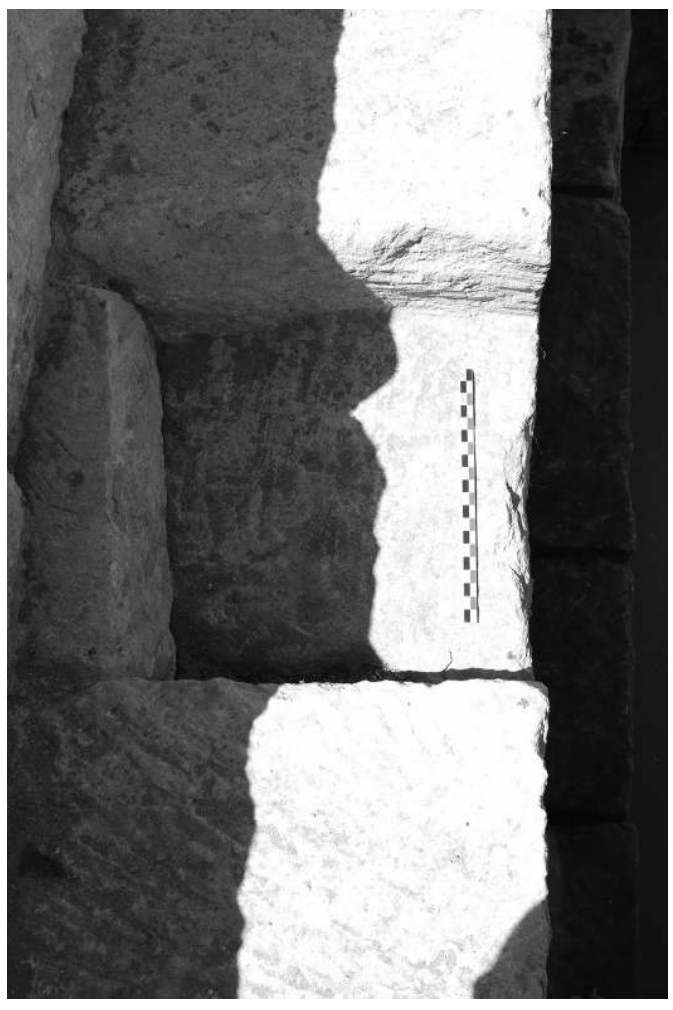

C. Castres / EFA. 


\section{Le matériel (fig. 15-17)}

Le matériel recueilli, pour l'essentiel des tessons de céramique, peut être distingué selon deux catégories: la céramique des couches supérieures, et la céramique de l'US 1517.

La céramique des couches supérieures est présente au-dessus des structures (US 1501 à 1515). Le faciès est similaire à ce que l'on trouve dans les autres sondages. Le matériel est varié et les formes bien connues: ESA, Atl. 3 notamment. Il s'agit des couches de remblaiement et de spoliation des blocs. Il n'est pas rare d'y trouver des tessons tardifs $\left(\mathrm{IV}^{\mathrm{e}}-\mathrm{V}^{\mathrm{e}}\right.$ siècle de notre ère). La présence de deux monnaies très certainement romaines (selon le poids et la taille, peut-être du III $^{e}$ siècle: AM 3929-3930) montre que cette phase d'arrachement des blocs et de recouvrement avec de la terre venue d'ailleurs est contemporaine des US identifiées comme les phases artisanales tardives. La présence de fragments de bassins oxydés, non compris dans les couches pigmentées (comme nous l'avons constaté dans le sondage $C$ et $D$ notamment en 2015 et 2016) témoigne une nouvelle fois d'une activité liée au traitement de la couleur (enduit mural ?) dans la zone du bassin intérieur à l'époque où les principales structures sont déjà recouvertes.

La céramique de l'US 1517 est associé à l'UC 1005. Il s'agit d'une couche bien plus homogène, dont l'essentiel des fragments semblent dater des époques archaïque et classique. Seuls deux tessons peuvent abaisser la datation, mais le matériel provient sans doute d'un environnement étendu brassé par les flots et rejeté contre le parement du mur, puis prisonnier de la sédimentation sableuse. Il n'est pas exclu non plus que les deux tessons hellénistiques (soit $2 \%$ du total de la céramique fine diagnostique) soient liés à une intrusion résiduelle. Les amphores sont également en grand nombre et, là encore, se distinguent des US supérieures par leur relative homogénéité.

Fig. 15. Tessons diagnostiques de l'âge du Fer de l'US 1517.

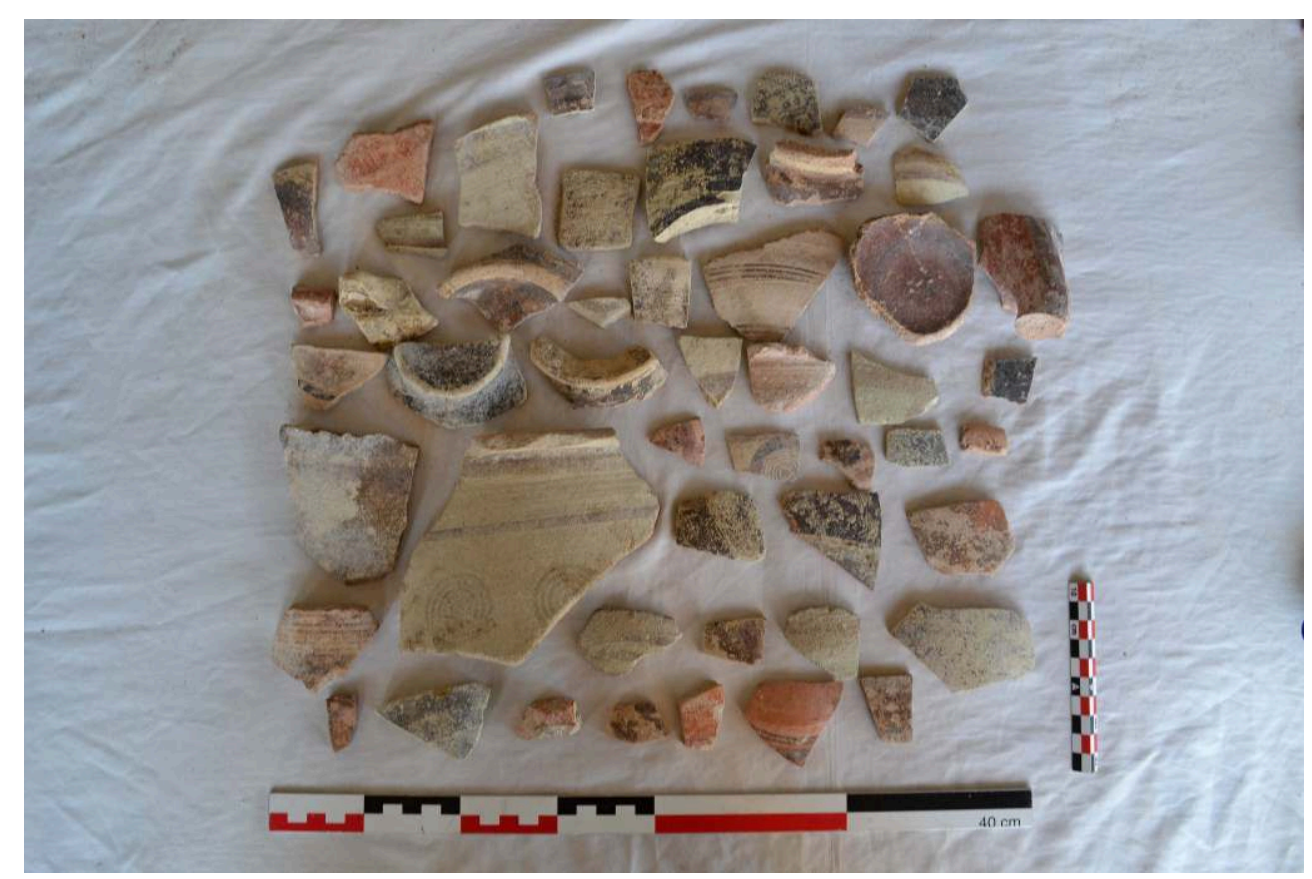

M. Hermsdorff / EFA. 
Fig. 16. Fragment de terre cuite chypro-archaïque.

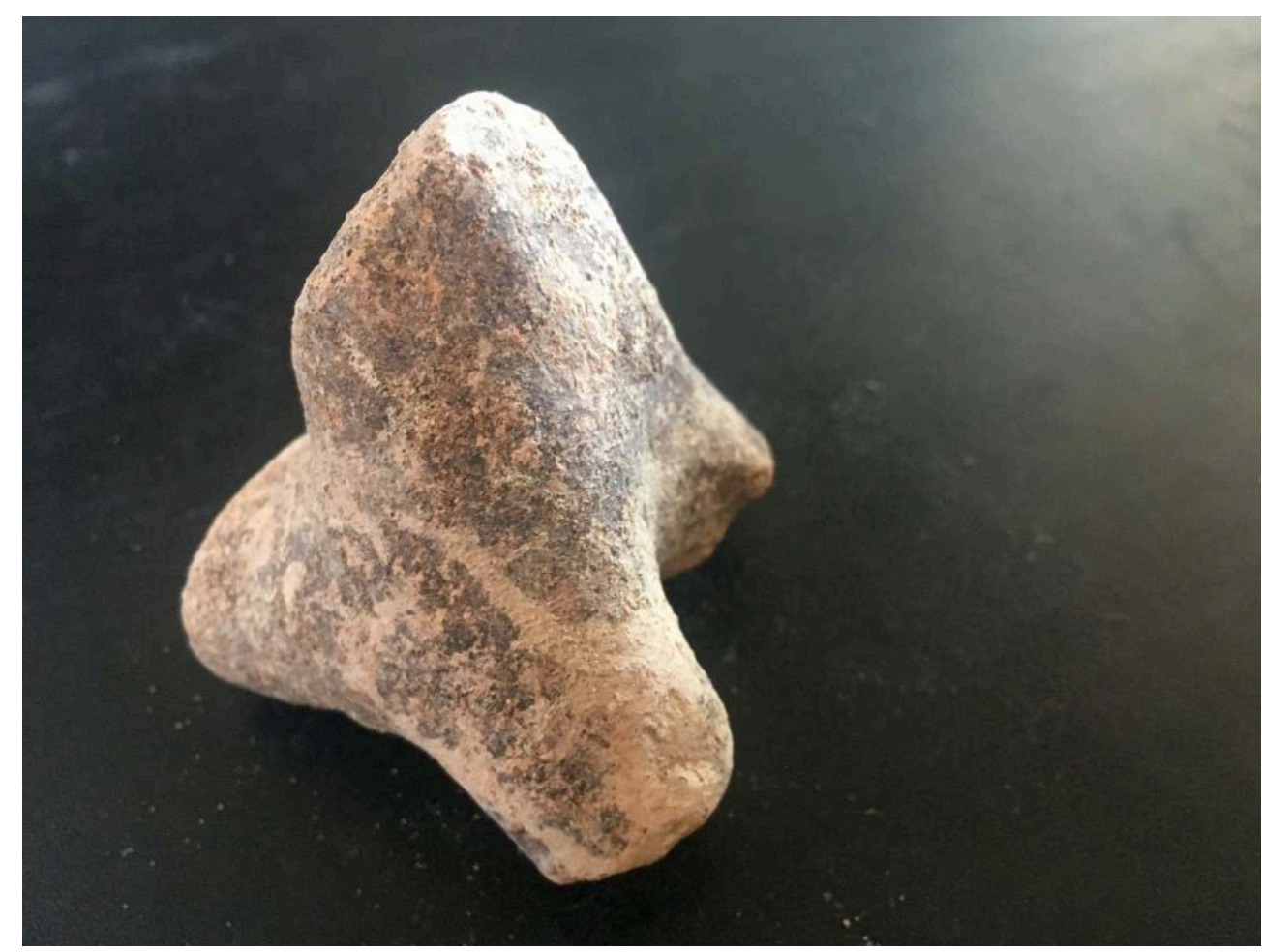

L. Thély / EFA.

\section{Identification et chronologie}

Les résultats obtenus à l'occasion de cette courte campagne paraissent exceptionnels à plusieurs titres.

D'abord d'un point de vue chronologique : si les couches supérieures ne présentent pas un caractère différent de celles qui recouvrent l'ensemble des structures mises au jour dans les autres sondages (ce qui témoigne d'une phase étendue de spoliation partout où les blocs proprement taillés ont pu être remployés), l'US 1517 jette un regard nouveau sur le phasage de cette partie de la zone urbanisée. Le sable a en effet tenu prisonnier un grand nombre de céramiques archaïques, dont les proportions sont clairement inversées par rapport aux autres couches. Il est très vraisemblable que les UC 1005 et 1012 aient été contemporains des phases les plus anciennes du site. Ce point est confirmé par l'excellente facture des constructions architecturales: aucun bloc en remploi, joints parfaitement réalisés et assises régulières en débord.

L'identification des structures mises au jour nécessite une certaine prudence, essentiellement due à la surface réduite fouillée à l'occasion de cette campagne. Néanmoins, il est certain que les structures UC 1005 et 1012 affectent une telle différence de construction par rapport aux autres murs de la zone qu'elles ne peuvent appartenir qu'à un ensemble fonctionnel différent.

Une hypothèse doit être considérée avec attention: celle de la présence de structures portuaires. L'ensablement progressif le long des structures témoigne que la mer se tenait à proximité. Les entrepôts, quoique plus tardifs, construits légèrement en 
surplomb (sans doute pour éviter d'être affectés par la salinité du milieu marin) pourraient être parfaitement en cohérence avec la présence d'un port plus ancien. En outre, la publication récente du port extérieur par J.-Y. Empereur ${ }^{3}$ précise que cet édifice militaire a été bâti peu avant 294 à l'occasion de la lutte entre Antigone et Ptolémée. Or, une cité de l'importance d'Amathonte ne peut avoir été dépourvue d'un port aux époques archaïque et classique. Les importations sont en si grand nombre depuis le Chypro-Géométrique qu'il est impossible qu'elles aient transité autrement que par un port. Enfin, des concrétions le long des assises inférieures, actuellement plongées dans l'eau, ont été prélevées : elles paraissent indiquer la mise en contact des structures avec de l'eau.

Le mode de construction de l'UC 1005, qui s'étage en degrés plus ou moins régulier, évoque plus précisément le système d'une cale sèche, qui servait à la réfection des navires. Si les parallèles antiques sont très rares et nécessitent une recherche approfondie, le modèle perdure encore de nos jours. Les degrés servent en effet à appuyer des madriers qui permettent le calage des bateaux. En outre, un carottage d'une profondeur de $1,20 \mathrm{~m}$ a été réalisé dans la zone aujourd'hui inondé par la remontée de la nappe phréatique. Il a montré qu'il existait des différences dans la texture sableuse : le niveau le plus tassé pourrait correspondre à l'espace de circulation des bateaux que l'on tirait pour les mettre au sec.

\section{Conclusion}

La campagne 2018 a permis d'apporter de nouveaux éléments de compréhension et de datation de la zone urbaine (voire urbano-portuaire) située entre l'agora et la mer. La découverte de nouvelles structures, dont la technique de construction diffère du reste, associé à une stratigraphie en place permet d'avancer prudemment l'hypothèse d'un espace ouvert sur la mer, qui remonterait la chronologie de manière significative au moins à l'époque archaïque.

\section{BIBLIOGRAPHIE}

EMPEREUR (éd.) 2018

Jean-Yves Empereur (éd.), The Hellenistic Harbour of Amathus: Underwater Excavations, 1984-1986. Volume 2: Artefacts Found during Excavations, Athènes, École française d'Athènes, Études Chypriotes 20, 2018.

EMPEREUR, KOŽELJ 2018

Jean-Yves Empereur, Tony Koželj, The Hellenistic Harbour of Amathus: Underwater Excavations, 1984-1986. Volume 1: Architecture and History, Athènes, École française d'Athènes, Études Chypriotes 19, 2018.

THÉLY et al. 2015-2016

Ludovic Thély, Julien Adam, Camille Castres, Antoine Chabrol, François-Dominique Deltenre, 
Antigone Marangou, Cécile Rocheron, « Rapport sur les travaux de l'École française d'Athènes en 2014 et en 2015 : Amathonte. Les abords Sud-Ouest de l'agora », BCH 139-140, 2015-2016, p. 980-1016.

THÉLY et al. à paraître 1

Ludovic Thély, Julien Adam, Dominique Barcat, Camille Castres, François-Dominique Deltenre, Antigone Marangou, Cécile Rocheron, « Amathonte : les abords Sud-Ouest de l'agora, 2016 », Bulletin archéologique des Écoles françaises à l'étranger.

THÉLY et al. à paraître 2

Ludovic Thély, Julien Adam, Marie-Hélène Barrière, Camille Castres, François-Dominique Deltenre, Antigone Marangou, Rachel Nouet, Cécile Rocheron, « Amathonte : les abords SudOuest de l'agora, 2017 », Bulletin archéologique des Écoles françaises à l'étranger.

\section{NOTES}

1. THÉLY et al. 2015-2016; THÉLY et al. à paraître 1 et 2.

2. Chronique des fouilles en ligne notice $\mathrm{n}^{\circ} 5067$, mise en ligne le 10 septembre 2015, consultée le 29 juin 2020, https://chronique.efa.gr/?kroute=report\&id=5067; Chronique des fouilles en ligne notice $n^{\circ} 5427$, mise en ligne le 5 juillet 2016, consultée le 29 juin 2020, https://chronique.efa.gr/?kroute=report\&id=5427; THÉLY et al. 2015-2016, p. 984-987.

3. EMPEREUR, KOŽELJ 2018 ; EMPEREUR (éd.) 2018.

\section{INDEX}

Thèmes : EFA

chronologie https://ark.frantiq.fr/ark:/26678/pcrtpcAdBe2SFu

lieux https://ark.frantiq.fr/ark:/26678/pcrtrvSIfnFAUb

Année de l'opération : 2018

sujets https://ark.frantiq.fr/ark:/26678/pcrtVemT606YVG, https://ark.frantiq.fr/ark:/26678/ pcrtwh5icnwutJ, https://ark.frantiq.fr/ark:/26678/pcrt4HEc49rMp7, https://ark.frantiq.fr/ark:/ 26678/pcrtVFfTq3JlGu, https://ark.frantiq.fr/ark:/26678/pcrtfx4s0yvSLj, https://ark.frantiq.fr/ ark:/26678/pcrtQuZiT5nYY2, https://ark.frantiq.fr/ark:/26678/pcrtUtcfIvQKcB

\section{AUTEURS}

\section{LUDOVIC THÉLY}

Ministère de l'Europe et des Affaires étrangères

\section{JULIEN ADAM}

Université de Namur 


\section{CAMILLE CASTRES}

USR 3155 « Institut de recherche sur l'architecture antique (IRAA »

ANTIGONE MARANGOU

Université de Rennes 2

CÉCILE ROCHERON

UMR 8546 « Archéologie et Philologie d'Orient et d'Occident (AOROC) » 